\title{
Coupled systems with hyperchaos and quasiperiodicity
}

\author{
Alexander P. Kuznetsov and Yuliya V. Sedova* \\ Kotel'nikov's Institute of Radio-Engineering and Electronics of RAS, Saratov Branch, \\ Zelenaya 38, Saratov, 410019, Russian Federation
}

(Dated: July 11, 2018)

\begin{abstract}
A model with hyperchaos is studied by means of Lyapunov two-parameter analysis. The regions of chaos and hyperchaos, as well as autonomous quasiperiodicity are identified. We discuss the picture of domains of different regimes in the parameter plane of coupled systems, corresponding to the cases of interaction of quasiperiodic and hyperchaotic subsystems.
\end{abstract}

PACS numbers: 05.45.-a, 05.45.Xt

A hyperchaotic attractor is characterized by the presence of several positive Lyapunov exponents. Dynamics of systems with hyperchaos is more complex and diverse than that of the system with simple chaos. Examples of hyperchaos were pointed out yet by Rössler and others researchers [1-6]. Recently some new suitable examples of models with hyperchaos were discovered. A study of such problems as scenarios of the emergence of hyperchaos, structure of the hyperchaotic attractor, a possibility of hidden attractors et al. was undertaken in refs. [7-11]. It should be noted that such systems are promising from the point of view of secure communication because they can realize high-dimensional chaos. However, many questions in the theory of hyperchaos remain open. For example, the problem of the interaction of the hyperchaotic systems is important and promising. In this report we discuss some aspects of this problem.

Let us consider a model suggested recently in paper [7]. It is a modified form of well-known Lorenz system:

$$
\begin{aligned}
& \dot{x}=a(y-x)+e y z, \\
& \dot{y}=c x-d x z+y+u, \\
& \dot{z}=x y-b z, \\
& \dot{u}=-k y .
\end{aligned}
$$

Here $x, y, z, u$ are the dynamical variables and $a, b, c, d, e, k$ are control parameters.

The model (11) has several fundamental properties and advantages [7]:

1. it is dissipative at $(a+b-1)>0$;

2. equations (11) are symmetric with respect to z-axis: $(x, y, z, u) \rightarrow(-x,-y, z,-u)$;

3. it has only the trivial zero equilibrium.

For fixed parameter values $a=35, c=25, d=5, e=$ $35, k=100$ Chen et al. [7] undertook the one-parameter analysis of model (11). In Fig. 1 we reproduced the spectrum of Lyapunov exponents and typical phase portraits

${ }^{*}$ Corresponding author.

Tel.: +7 8452278685; fax: +7 8452272401 .

E-mail address: sedovayv@yandex.ru at varying parameter $b$. Note that, as usual, one exponent is always zero $\Lambda_{3}=0$ and is not shown in the figure.
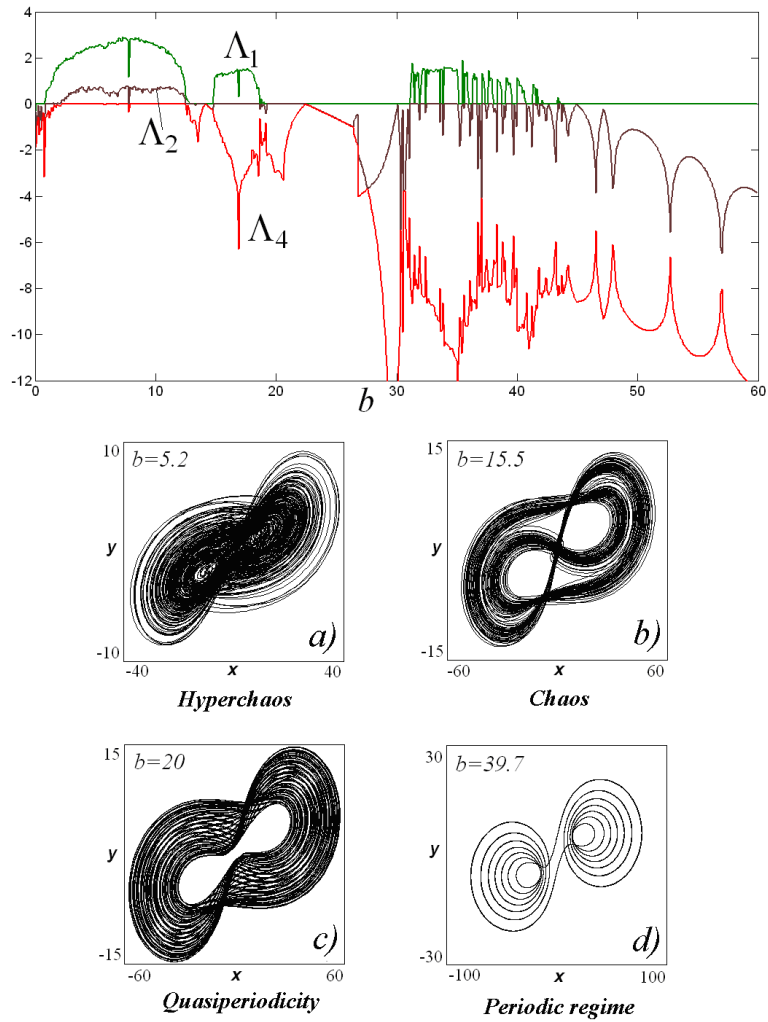

FIG. 1: Plot of the Lyapunov exponents as a function of parameter $b$ for the model (11). (Exponent $\Lambda_{3}=0$ is not shown in the plot.)

The system (11) exhibits hyperchaos in a wide range of parameter values. It is interesting that along with such a regime, as well as periodic and chaotic regimes, the model also demonstrates quasiperiodic dynamics. Thus, model (11) is one more example of an autonomous system with quasiperiodicity (see. also $[13,14]$ ); this fact increases its importance.

One-parameter analysis does not give us full information about typical regimes and their localization. That is why we turn to the two-parameter analysis. Let us use 
charts of Lyapunov exponents [15-17]. We calculate the values of the Lyapunov exponents at each point of the plane $(k, b)$ and color this plane in accordance with the type of observed regime:

- periodic regime $P$ (all exponents are negative);

- quasiperiodic regime $T_{2}$ (one zero exponent);

- chaos $C$ (one positive exponent);

- hyperchaos $H C$ (two positive exponents).

(One trivial zero exponent is not taken into consideration.) The corresponding chart of Lyapunov exponents is shown in Figure 2. The color palette is presented to the right of the figure. It can be seen that the area of hyperchaos entirely occupies extensive part of the plane of the control parameters. The domain of existence of twofrequency tori is visualized very well. We can distinguish the boundary of quasiperiodicity region - the NeimarkSacker bifurcation line with the Arnold tongues emerging from it.

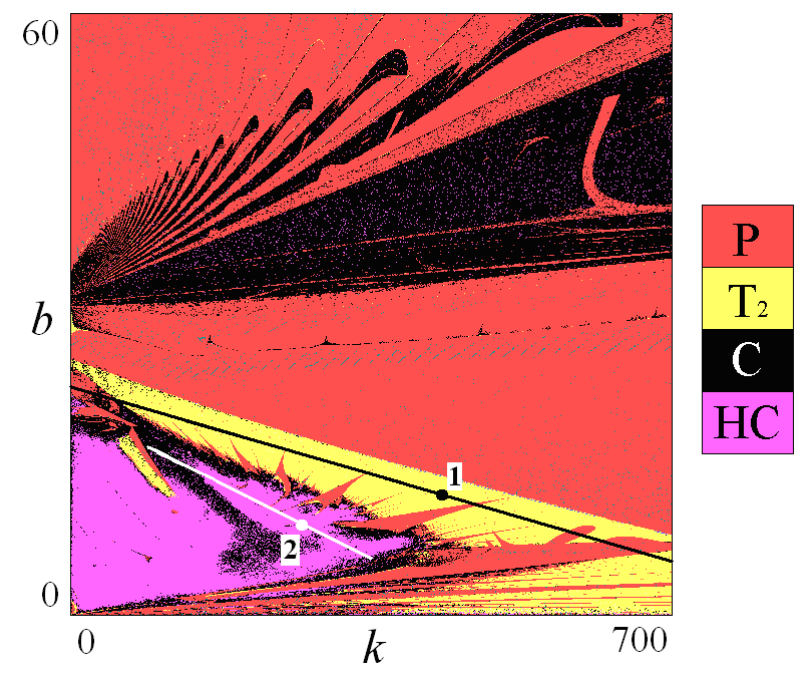

FIG. 2: Chart of Lyapunov exponents of the system (1). The color corresponds to the type of regime. Two lines are shown for which the scan of parameter plane is executed.

Let us study now dynamics of two coupled models (1), the coupling terms are represented by the differences of the corresponding variables:

$$
\begin{aligned}
& \dot{x}_{1}=a\left(y_{1}-x_{1}\right)+e y_{1} z_{1}+\mu\left(x_{2}-x_{1}\right), \\
& \dot{y}_{1}=c x_{1}-d x_{1} z_{1}+y_{1}+u_{1}+\mu\left(y_{2}-y_{1}\right), \\
& \dot{z}_{1}=x_{1} y_{1}-b_{1} z_{1}+\mu\left(z_{2}-z_{1}\right) \\
& \dot{u}_{1}=-k_{1} y_{1}+\mu\left(u_{2}-u_{1}\right) \\
& \dot{x}_{2}=a\left(y_{2}-x_{2}\right)+e y_{2} z_{2}+\mu\left(x_{1}-x_{2}\right), \\
& \dot{y}_{2}=c x_{2}-d x_{2} z_{2}+y_{2}+u_{2}+\mu\left(y_{1}-y_{2}\right), \\
& \dot{z}_{2}=x_{2} y_{2}-b_{2} z_{2}+\mu\left(z_{1}-z_{2}\right) \\
& \dot{u}_{2}=-k_{2} y_{2}+\mu\left(u_{1}-u_{2}\right) .
\end{aligned}
$$

Here $x_{1}, y_{1}, z_{1}, u_{1}$ and $x_{2}, y_{2}, z_{2}, u_{2}$ are dynamical variables of the first and the second subsystems, respectively, and $\mu$ is the coupling parameter. We fixed a set of parameters $a=35, c=25, d=5, e=35$ and change coefficients $k_{1}, k_{2}, b_{1}, b_{2}$.

The presence of set of Arnold tongues of different periods in the autonomous system allows us to associate with the model the so-called "frequency parameter", corresponding to the motion along the Neimark-Sacker bifurcation line. We choose two routes corresponding to varying of this effective parameter value - one lies through the region of quasiperiodic regimes characterized by built-in set of Arnold tongues (Route 1 in Figure 2) and the second through the area of hyperchaos (Route 2 ).

These routes correspond to variation of the frequency parameter in the second subsystem. In the first subsystem let us fix the values $k_{1}, b_{1}$ so that we can observe either quasiperiodicity or hyperchaos. The values of these fixed parameters are marked by points on lines in Figure 2 .

The first scenario. Let us fix parameters $k_{1}=430$, $b_{1}=12$ in the first subsystem and plot a chart of Lyapunov exponents. Herewith along the $\mathrm{y}$-axis we will vary coupling parameter $\mu$ and along x-axis - coefficient $k_{2}$ measured along the marked Route 1 in Figure 2. This case corresponds to interaction of quasiperiodic oscillations. The resulting chart of Lyapunov exponents is displayed in Figure 3. Now we have an additional possibility of the presence of a three-frequency tori $T_{3}$ (two zero exponents) and four-frequency tori $T_{4}$ (three zero exponents).

At a low coupling parameter of the model (2) it is naturally to expect an emergence of four-frequency torus - this hypothesis is confirmed by the numerical study. Also on the chart we can distinguish wide area of twofrequency tori in form of peaked tongue touching the line of zero coupling. The point of contact corresponds to the condition $k_{2}=k_{1}, b_{2}=b_{1}$ when the subsystems are identical and their "frequency parameters" coincide. Also, there are narrow tongues of $T_{3}$ torus embedded in the region of $T_{4}$ tori. In right part of Figure 3 we demonstrate the phase portraits and Poincaré sections at points of presence of $T_{2}, T_{3}$ and $T_{4}$ tori. A very narrow bands of periodic regimes are visualized.

Note that inside the area of two-frequency tori it is possible to observe a phase synchronization - some kind of synchronous quasiperiodicity. Illustrations of such a regime are given in Fig.4. We display the phase portraits of both partial systems of model (2), for which it is possible to determine the phase of the oscillations (Figure $4 \mathrm{a}, \mathrm{b})$. Fig.4c represents the time-dependence of the phase difference $\varphi_{1}-\varphi_{2}$; phase is calculated according to the formula $\varphi_{1}=\arctan \left(y_{1} / x_{1}\right), \varphi_{2}=\arctan \left(y_{2} / x_{2}\right)$. It can be seen that the phase difference $\varphi_{1}-\varphi_{2}$ changes over time in a limited range, which means the phase synchronization. Another proof of the existence of phase synchronization is a specific form of the phase diagram in Fig.4d. In Fig.4e we have shown time-dependence of 

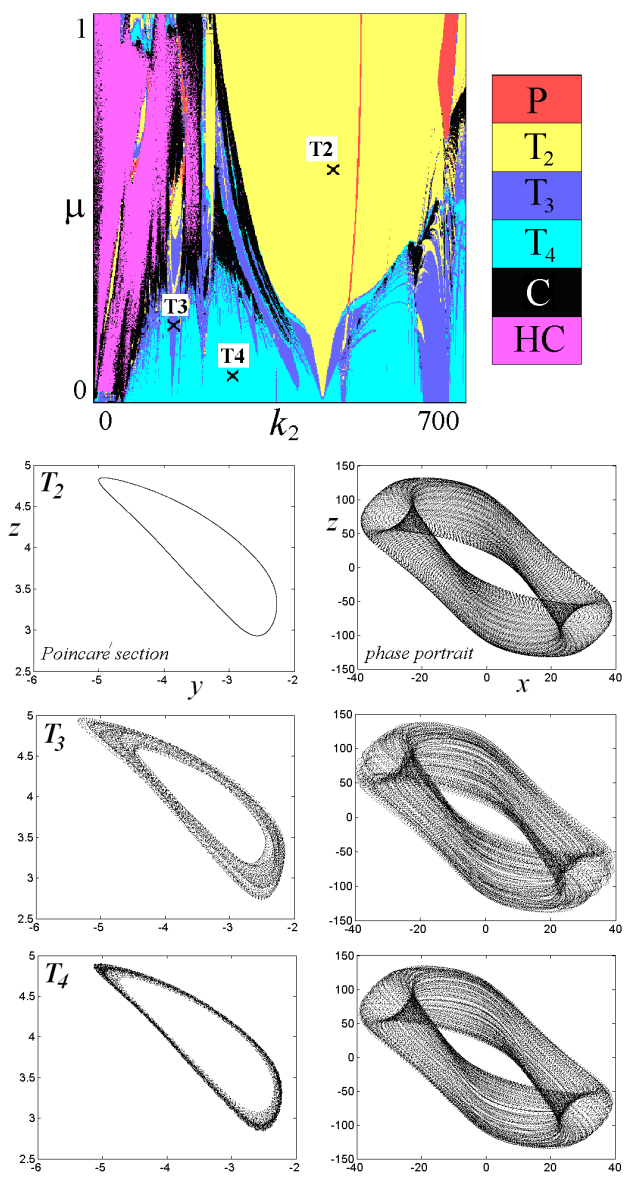

FIG. 3: Chart of Lyapunov exponents for the system (2), $a=$ $35, c=25, d=5, e=35, k_{1}=430, b_{1}=12$. Parameters $k_{2}$, $b_{2}$ are varied along the Route 1 in Figure 2. Poincaré sections and phase portraits in the selected points on the Lyapunov chart are presented on the lower part of the Figure.

the first and second subsystems amplitudes $R_{1}$ and $R_{2}$.

The second scenario. Now in the second subsystem we will vary the parameters along the Route 2 in Figure 2. The first subsystem generates hyperchaotic oscillations; we choose the selected point $k_{1}=430, b_{1}=12$ on this line. Thus, we are going to discuss the case of interaction of two subsystems with hyperchaos. Since every subsystem has two positive exponents in their own Lyapunov spectrum, on Lyapunov chart it is logical to expect the area $\mathrm{HC} 4$ with four positive Lyapunov exponents. This idea is confirmed by Fig.5a, that demonstrates regimes with two, three and four positive Lyapunov exponents. It is remarkable, that at large coupling not only the number of positive Lyapunov exponents is reduced, but the regions of two-frequency quasiperiodicity (although very narrow) arise. Thus, the interaction of the hyperchaotic subsystems can lead to quasiperiodicity. This statement is illustrated in Fig.5b, which presentes an enlarged fragment of the Lyapunov chart. We can see the area of quasiperiodic oscillations and built-in system of Arnold tongues.
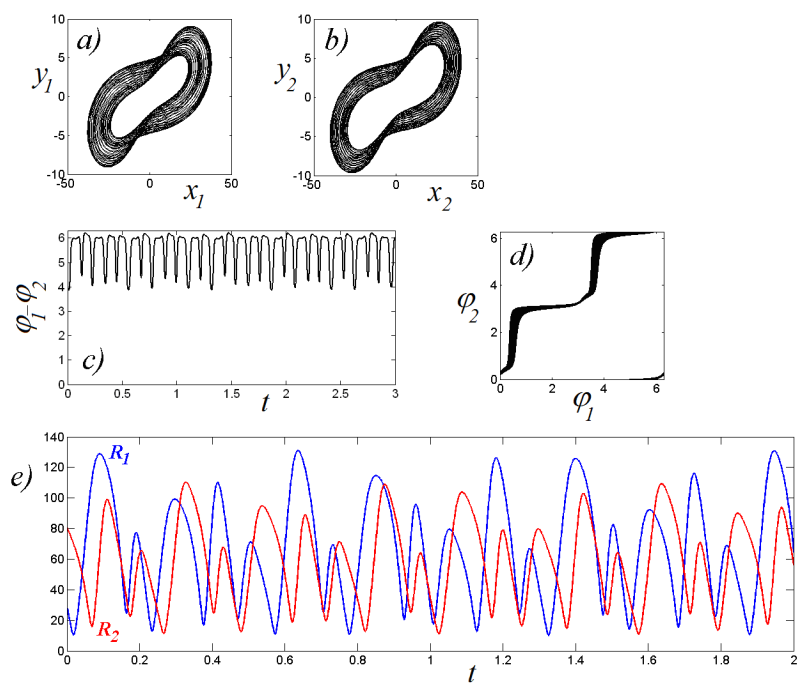

FIG. 4: Illustrations of synchronous quasiperiodicity, $k_{2}=$ $320, \mu=0.7$. a), b) - phase portraits of both subsystems, c) the time-dependence of the phase difference, d) phase diagram, e) the time-dependence of subsystem amplitudes.
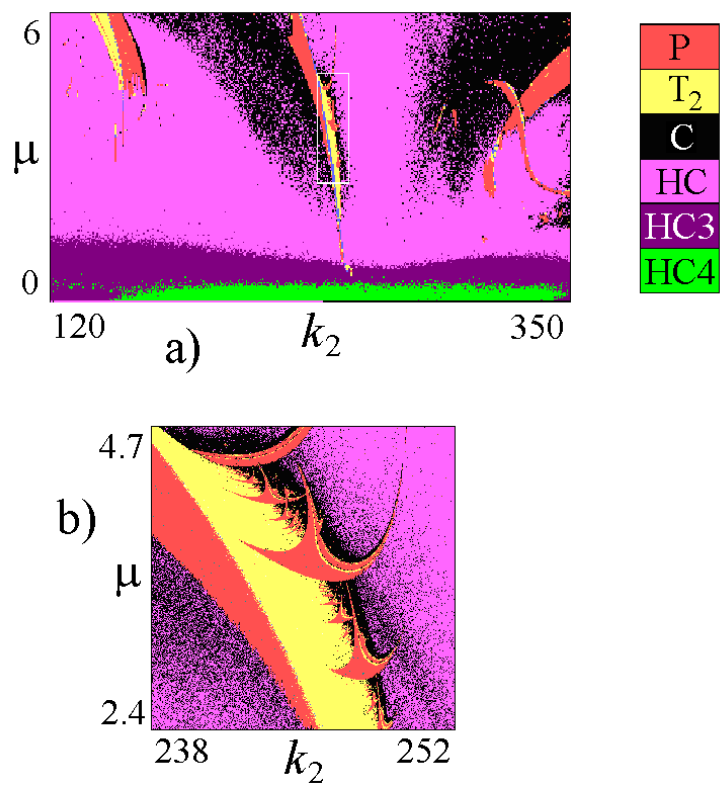

FIG. 5: The chart of Lyapunov exponents of model (2) and its enlarged fragment.

Figure 6 is a plot of the largest four Lyapunov exponents along the line $k_{2}=$ const in Fig.5a. An interesting point is a noticeable change in the irregularity of the plots in comparison with Fig.1. Thus, hyperchaos arising from the interaction of subsystems is rather rough, i.e. weakly dependent on parameters. This fact may be important for applications.

Thus, in the present report we deal with the problems of the interaction of the subsystems that can exhibit quasiperiodicity and hyperchaos in autonomous mode. 


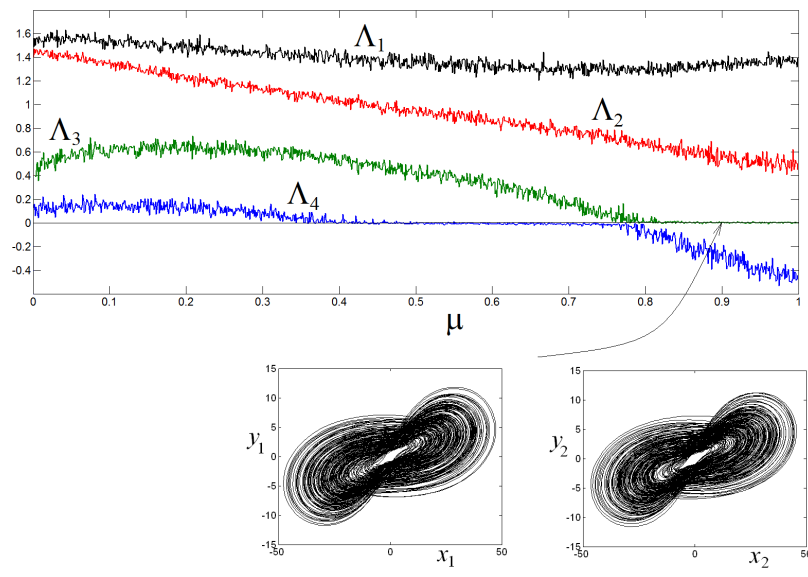

FIG. 6: Plot of the largest Lyapunov exponents as a function of the coupling parameter along the line $k_{2}=285$ in Fig.5. Phase portraits at $\mu=0.9$ are presented.
For coupled subsystems with autonomous quasiperiodicity we detect the regime of synchronous quasiperiodicity. One of interesting results is the emergence of quasiperiodic oscillations for the interacting hyperchaotic systems.

This research was supported by the Grant of RF President program for leading Russian research school NSh1726.2014.2 and Russian Foundation for Basic Research grant 14-02-00085.
[1] O.E. Rossler, An equation for hyperchaos, Physics Letters A 71(2-3) (1979) 155-157.

[2] M. Klein, G. Baier, O.E. Rössler, From N-tori to hyperchaos, Chaos, Solitons \& Fractals, 1(2) (1991) 105-118.

[3] E. Barreto, B.R. Hant, C. Grebogi, J.A. Yorke. From high dimensional chaos to stable periodic orbits: The structure of parameter space, Physical Review Letters 78 (1997) 4561-4564.

[4] M.A. Harrison, Y.-C. Lai, Route to high-dimensional chaos, Physical Review E 59 (1999) 3799-3802.

[5] T. Kapitaniak, Yu. Maistrenko, S. Popovych, Chaoshyperchaos transition, Physical Review E 62 (2000) 19721976.

[6] H. Richter, The generalized Hénon maps: examples for higher-dimensional chaos, International Journal of Bifurcation and Chaos 12(06) (2002) 1371-1384.

[7] Z. Chen, Y. Yang, G. Qi, Z. Yuan, A novel hyperchaos system only with one equilibrium, Physics Letters A 360(6) (2007) 696-701.

[8] K.P. Harikrishnan, R. Misra, G. Ambika, On the transition to hyperchaos and the structure of hyperchaotic attractors, The European Physical Journal B 86(9) (2013) $1-12$.

[9] X. Han, B. Jiang, Q. Bi, 3-torus, quasi-periodic bursting, symmetric subHopf/fold-cycle bursting, subHopf/foldcycle bursting and their relation, Nonlinear Dynamics 61(4) (2010) 667-676.

[10] W. Wu, Z. Chen, Z. Yuan, The evolution of a novel fourdimensional autonomous system: among 3-torus, limit cycle, 2-torus, chaos and hyperchaos. Chaos, Solitons \&
Fractals 39(5) (2009) 2340-2356.

[11] Z. Wei, W. Zhang, Hidden hyperchaotic attractors in a modified Lorenz-Stenflo system with only one stable equilibrium, International Journal of Bifurcation and Chaos 24(10) (2014) 1450127 .

[12] A. Pikovsky, M. Rosenblum, J. Kurths, Synchronization. A Universal Concept in Nonlinear Sciences, Cambridge University Press, 2001.

[13] A.P. Kuznetsov, S.P. Kuznetsov, N.V. Stankevich, A simple autonomous quasiperiodic self-oscillator, Communications in Nonlinear Science and Numerical Simulation 15(6) (2010) 1676-1681.

[14] V. Anishchenko, S. Nikolaev, J. Kurths, Peculiarities of synchronization of a resonant limit cycle on a twodimensional torus, Physical Review E 76(4) (2007) 046216.

[15] C. Baesens, J. Guckenheimer, S. Kim, R.S. MacKay, Three coupled oscillators: mode locking, global bifurcations and toroidal chaos, Physica D: Nonlinear Phenomena 49(3) (1991) 387-475.

[16] Yu.P. Emelianova, A.P. Kuznetsov, I.R. Sataev, L.V. Turukina, Synchronization and multi-frequency oscillations in the low-dimensional chain of the self-oscillators, Physica D: Nonlinear Phenomena 244(1) (2013) 36-49.

[17] Y.P. Emelianova, A.P. Kuznetsov, L.V. Turukina, I.R. Sataev, N.Yu. Chernyshov, A structure of the oscillation frequencies parameter space for the system of dissipatively coupled oscillators, Communications in Nonlinear Science and Numerical Simulation 19(4) (2014) 12031212 . 